\title{
In vivo meiotic resumption, fertilization and early embryonic development in the bitch
}

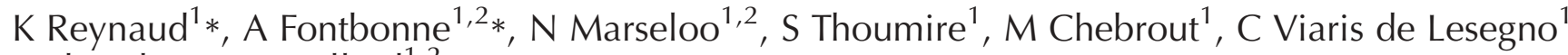 \\ and S Chastant-Maillard ${ }^{1,2}$ \\ ${ }^{1}$ UMR 1198 INRA/ENVA Biologie du Développement et Reproduction and ${ }^{2}$ UP Reproduction, CERCA (Centre \\ d'Etudes en Reproduction des Carnivores), Ecole Nationale Vétérinaire d'Alfort, 7 Avenue du Général de Gaulle, \\ 94704 Maisons-Alfort Cedex, France
}

Correspondence should be addressed to K Reynaud; Email: kreynaud@vet-alfort.fr

*(K Reynaud and A Fontbonne contributed equally to this work)

\begin{abstract}
Early development in canine species follows a very specific pattern. Oocytes are ovulated at the germinal vesicle stage and meiotic resumption occurs in the oviduct. However, because of difficulties in the accurate determination of ovulation time and in the observation of oocyte nuclear stage by light microscopy, these early events have not been fully described. Moreover, the oocyte stage at which sperm penetration occurs is still uncertain since fertilization of immature oocytes has been reported in vivo and in vitro. The aim of this study was to establish the exact timing of in vivo meiotic resumption, fertilization and early embryo development in the bitch with reference to ovulation. Ovulation was first determined by ultrasonography, artificial inseminations were performed daily and oocytes/embryos were collected between 17 and $138 \mathrm{~h}$ after ovulation. After fixation and DNA/tubulin staining, the nuclear stage was observed by confocal microscopy. Of the 195 oocytes/embryos collected from 50 bitches, the germinal vesicle stage was the only one present until $44 \mathrm{~h}$ post-ovulation, and the first metaphase II stage was observed for the first time at $54 \mathrm{~h}$. Sperm penetration of immature oocytes appeared to be exceptional (three out of 112 immature oocytes). In most cases, fertilization occurred from $90 \mathrm{~h}$ post-ovulation in metaphase II oocytes. Embryonic development was observed up to the eight-cell stage. No significant influence of bitch breed and age on ovulation rate, maturation and developmental kinetics was observed. However, some heterogeneity in the maturation/development process was observed within the cohort of oocytes/embryos collected from one bitch. In conclusion, the most peculiar aspect of the canine species remains oocyte meiotic maturation whereas fertilization follows the same pattern as in other mammals.
\end{abstract} Reproduction (2005) 130 193-201

\section{Introduction}

Among mammals, the canine species is well known for its unusual pattern of oocyte meiosis: at ovulation, an oocyte is released in the germinal vesicle (GV) stage and meiotic resumption occurs after about $48 \mathrm{~h}$ spent in the oviduct. Moreover, the endocrine environment at ovulation in the bitch, is highly different to that observed in other species, since follicles undergo a preovulatory luteinization after the luteinizing hormone $(\mathrm{LH})$ peak and serum progesterone has already reached high levels at ovulation.

The kinetics of meiosis and embryonic development with reference to ovulation has not, however, been fully described. The precise determination of this chronology is very important for canine reproductive biotechnologies, especially to select the optimal moment for artificial insemination with frozen semen or embryo transplantation. In previous studies, the time of ovulation has not been precisely defined and embryonic development was either assessed in respect to the moment of the LH peak or the progesterone level, or was observed by laparoscopy (Tsutsui \& Shimizu 1975, Archbald et al. 1980, Bysted et al. 2001). Secondly, determination of the nuclear stage in canine oocytes by stereomicroscopy without DNA staining (Tsutsui \& Shimizu 1975) or fluorescent optic microscopy (Saint-Dizier et al. 2004) is quite difficult and uncertain, because of the high lipid content of their cytoplasm. Nowadays, advanced observation techniques such as immunocytochemistry coupled with confocal microscopy are available and have been shown to be especially effective in the observation of the bitch oocyte (Reynaud et al. 2004, Saint-Dizier et al. 2004). Nevertheless, the exact timing of the oocyte maturation step remains to be determined, as well as the exact role of sperm in meiotic resumption. Indeed, sperm heads have already been observed in vivo in immature bitch oocytes 
(Van der Stricht 1923, fox: Farstad et al. 1993). In vitro, this penetration at an immature stage has been shown to occur with a high frequency and to act as an inducer of meiotic resumption (Saint-Dizier et al. 2001).

The aims of this study were thus to evaluate the precise kinetics of oocyte nuclear maturation as well as early embryonic development in the bitch, and to examine whether sperm penetration at immature stages is a physiological feature in vivo. Ovulation time was accurately determined through ovarian ultrasonography, and nuclear stages were defined by confocal microscopy after DNA/tubulin staining.

\section{Materials and Methods}

\section{Animals}

Fifty bitches, aged 8 months to 9 years (mean $3.80 \pm 0.34$ years), were included in this study. Twenty-two were Beagles $(10-13 \mathrm{~kg})$ from our research kennel and 28 were mongrel or pure-bred bitches from owners and breeders (3-40 kg) attending a consultation for routine ovariectomy. The protocol was approved by the Ethics committee of the National Veterinary College of Alfort.

\section{Oestrus detection and determination of ovulation time}

Ovarian cycles of the bitches were followed weekly by performing vaginal smears (for our experimental kennel bitches) and observing vulvar bleeding to detect onset of the heat period. During heat, serum progesterone levels were assayed every day. The progesterone assay was performed using an Elecsys kit (Roche Diagnostics, Meylan, France). When the concentration started to increase above $2 \mathrm{ng} / \mathrm{ml}$, ovarian ultrasonography was performed two to three times a day (ultrasonograph HDI 3500, probe 7.5 MHz; ATL, Phillips Systèmes Médicaux, Suresnes, France). Before ovulation, follicles were identified as anechogenic structures (starting from $1 \mathrm{~mm}$ in diameter and reaching 5-7 $\mathrm{mm}$ just before ovulation). At the time of ovulation, the follicular anechogenic cavity disappears or its diameter dramatically decreases $(2-3 \mathrm{~mm})$ with the inner follicular wall becoming fuzzy. Furthermore, liquid is often visible surrounding the ovary (Hayer et al. 1993). This process lasts for a few hours and therefore the reference to ovulation was set at the mean time between the last ultrasonography with all follicles visible and the one where there was a significant change or complete disappearance of follicles.

\section{Artificial insemination}

To allow eventual penetration of spermatozoa in immature oocytes, the permanent presence of sperm in the oviducts was maintained starting before ovulation. Intra-uterine artificial inseminations using a Scandinavian catheter (Andersen 1975) were thus performed once a day from the day before ovulation (progesterone level around $2-3 \mathrm{ng} / \mathrm{ml}$, large follicles $>4 \mathrm{~mm}$ ) to the day of ovariectomy. Semen was collected daily from two Beagle males with known high in vivo fertility, using manual stimulation. After evaluation of the concentration and mobility, the two ejaculates were pooled and fresh semen was used for uterine insemination (spermatic and prostatic fractions).

\section{Collection and fixation of canine oocytes and embryos}

Ovariectomies were performed using a conventional surgical procedure from 15 to $136 \mathrm{~h}$ after ovulation. Ovaries, oviducts and the tip of the uterine horns were immediately collected and kept at $38{ }^{\circ} \mathrm{C}$. The number of corpora lutea and non-ovulated follicles was evaluated and oviducts were carefully dissected from the fat of the ovarian bursa. A cannula 3.5 French (Tom Cat catheter; Kendall, Coveto, Montaigu, France) was inserted into the distal ostium of the oviduct (when surgery was performed during the first $24 \mathrm{~h}$ after ovulation) or in the infundibulum ( $24 \mathrm{~h}$ post-ovulation). Oviducts were flushed with $20 \mathrm{ml}$ warm medium 199 (M199; Sigma, St Quentin-Fallavier, France) supplemented with $20 \%$ fetal calf serum (FCS; Invitrogen, Cergy Pontoise, France). Oocytes/embryos were immediately fixed at $38{ }^{\circ} \mathrm{C}$ in $2 \%$ paraformaldehyde (Merck, Fontenay-sous-bois, France; w/v in phosphate-buffered saline (PBS) from Sigma) for $30 \mathrm{~min}$, and then in $4 \%$ paraformaldehyde ( $\mathrm{w} / \mathrm{v}$ in PBS) for a further $30 \mathrm{~min}$. They were rinsed in $\mathrm{PBS}+1 \%$ fraction $\mathrm{V}$ BSA (bovine serum albumin; Sigma). The diameter of the oocyte was measured (zona pellucida excluded; $\times 400$; IX70 inverted microscope; Olympus, Rungis, France) and oocytes were then stored at $4{ }^{\circ} \mathrm{C}$ until immunocytochemistry.

Flushing medium was centrifuged ( 5 min; $200 \mathrm{~g}$ ) and the presence of spermatozoa was evaluated in the pellet by optical microscopy ( $\times$ 400; microscope BX41; Olympus).

\section{Determination of maturation or developmental stage of oocytes/embryos}

When numerous granulosa cells were still present around the oocytes, decoronization was performed by gentle pipeting or incubation in $0.7 \%$ pronase for $45 \mathrm{~s}$ (Roche; w/v in M199) (Reynaud et al. 2004).

Microtubule visualization was performed by immunocytochemistry according to the technique used in the rabbit (Adenot et al. 1997). Briefly, after fixation and three washes in PBS, oocytes were incubated in a blocking solution (PBS containing 20\% FCS and 0.5\% Triton X-100) for $30 \mathrm{~min}$ at $37^{\circ} \mathrm{C}$, then incubated for $60 \mathrm{~min}$ at $37^{\circ} \mathrm{C}$ with mouse $\alpha$-tubulin monoclonal antibody (Sigma) diluted 1:200 in PBS/2\% FCS/0.5\% Triton. After three washes in $\mathrm{PBS} / 2 \% \mathrm{FCS} / 0.5 \%$ Triton, oocytes were incubated for $60 \mathrm{~min}$ at $37^{\circ} \mathrm{C}$ in Alexa fluor-conjugated rabbit anti-mouse antibody + ethidium homodimer-2 (Molecular Probes, Interchim, Asnières, France) diluted 1:500 in $\mathrm{PBS} / 2 \% \mathrm{FCS} / 0.5 \%$ Triton (final concentration of $4 \mu \mathrm{g} / \mathrm{ml}$ and $2 \mu \mathrm{M}$ respectively). Stained oocytes were then mounted on slides with Vectashield (Vector Laboratories, 
Interchim, Asnières, France). The slides were examined using confocal laser scanning microscopy (CLSM 310 Carl Zeiss, Gottingen, Germany; excitation wavelength 488 $\mathrm{nm})$. Oocytes were individually classified according to chromatin configuration as GV, germinal vesicle breakdown (GVBD), metaphase I and II (MI and MII), telophase I and II (TI and TII), 'picnotic DNA' and 'unidentifiable nuclear material' as described in Saint-Dizier et al. (2004). The presence of sperm heads in oocyte cytoplasm and in zona pellucida was also noted. For embryos, the number of cell nuclei was counted.

\section{Examination of $G V$ structure}

\section{Lamin detection}

After fixation, oocytes were sectioned into two parts using a scalpel blade in order to allow antibody penetration, and then incubated in PBS/2\% BSA for $15 \mathrm{~min}$. Incubation with the primary antibody (anti-lamin B1 goat polyclonal antibody; Santa Cruz Biotechnology, Santa Cruz, CA, USA) at 1/200 dilution was allowed to proceed overnight at $4{ }^{\circ} \mathrm{C}$ before two washes with $\mathrm{PBS} / 0.5 \%$ Tween 20 and two washes with PBS (10 min each). Oocytes were then incubated with the secondary antibody (FITC-conjugated anti-goat antibody; Jackson ImmunoResearch Laboratories, Interchim, France) at $1 / 300$ dilution for $1 \mathrm{~h}$ at room temperature. DNA was then stained for $15 \mathrm{~min}$ with ethidium homodimer-2 (2 $\mu \mathrm{M}$ in PBS/BSA; Molecular Probes) and oocytes were post-fixed in $2 \%$ paraformaldehyde $(15 \mathrm{~min})$. For confocal observation, oocytes were mounted on slides in Vectashield.

\section{Semi-thin sections}

Cumulus-oocyte complexes were fixed in 3\% glutaraldehyde/0.2 M sodium cacodylate/PBS for $90 \mathrm{~min}$, post-fixed in a solution of $2 \%$ osmium tetraoxide $/ 3 \%$ potassium ferrocyanide in $0.1 \mathrm{M}$ sodium cacodylate/PBS for $60 \mathrm{~min}$, dehydrated in a graded series of ethanol solutions $(30-100 \%)$ and embedded in Epon. Semi-thin sections $(2 \mu \mathrm{m})$ were cut using an ultramicrotome (Reichert E; Leica Microsystems, Rueil-Malmaison, France) and stained in a solution of $1 \%$ methylene blue and $1 \%$ azur II in $1 \%$ borax.

\section{Statistical analysis}

Influences of breed (Beagle vs others) and age on maturation and developmental kinetics were tested using Chisquare test. Variance analysis (general linear model procedure; SAS 1992) was performed to test the influence of bitch breed, age and their interaction on ovulation rate. Pearson's linear correlation test was used to evaluate the relationship between age and number of corpora lutea. All data are presented as means \pm S.E.M. The level of statistical significance was $P=0.05$.

\section{Results}

\section{Collection of canine oocytes/embryos}

A mean total of $7.89 \pm 0.57$ corpora lutea $(2-22 ; n=46$ bitches) was detected in the ovaries. There were no significant influence of bitch age, breed and no interaction between age and breed. The number of corpora lutea was significantly higher on the right compared with the left ovary $(4.22 \pm 0.40$ and $3.15 \pm 0.30$ respectively; $P<0.05)$. Non-ovulated follicles, defined as structures full of serous liquid, larger than $5 \mathrm{~mm}$ were observed in 12 bitches (32\%). For these bitches, between one and four non-ovulated follicles were observed per bitch, representing a mean of $23 \%$ (from 10 to $44 \%$ ) of the follicles present on both ovaries.

A total of 287 oocytes/embryos were collected from the 50 bitches $(5.74 \pm 0.48$ per bitch, from 0 to 19 maximum per bitch). Global recovery rate (number of structures collected/number of corpora lutea) was $72.4 \pm 3.9 \%$ varying from 0 to $150 \%$ according to the bitch. In two cases, the number of oocytes collected was found to be higher than the number of corpora lutea observed (respectively eight and six structures collected for seven and four corpora lutea). No dilatation of the oviduct was observed.

\section{Oocyte/embryo characteristics}

Mean oocyte diameter was $82.4 \pm 0.6 \mu \mathrm{m}(n=166$; from 58.8 to $100.7 \mu \mathrm{m}$ ) when measured without zona pellucida and $111.3 \pm 0.7 \mu \mathrm{m}(n=162$; from 86.2 to $131.5 \mu \mathrm{m})$ with the zona, when the limit between zona and granulosa cells was clearly defined. Some intra-bitch variability (maximum $20.4 \mu \mathrm{m}$ in the cohort of oocytes from the same bitch) was observed.

None of the cumulus-oocyte complexes presented mucification at any stage after ovulation (Fig. 1). Soon after ovulation (Fig. 1a), oocytes were surrounded by two to three layers of dense granulosa cells. Later on, even if the external layer became more loosely attached, the inner ones remained compact (Fig. 1b). Despite the presence of very dense granulosa cells, some immature oocytes were found to be fertilized (Fig. 1c: fertilized MI oocytes). During their stay in the oviduct, oocytes/embryos progressively lost the surrounding cells (Fig. 1d and e) but this denudation was not systematically observed (compare two- and eight-cell embryos in Fig. 1e and $\mathrm{f}$ respectively).

\section{Kinetics of meiotic resumption and early embryonic development}

Due to the high cytoplasmic lipid content, oocyte and early embryo stages are impossible to determine under light microscopy. Of the 43 bitches, 195 oocytes/embryos were stained and submitted to confocal microscopy. Representative examples of all stages observed are shown in Fig. 2. Maturation and developmental stages observed for each bitch are summarized in Fig. 3 . 

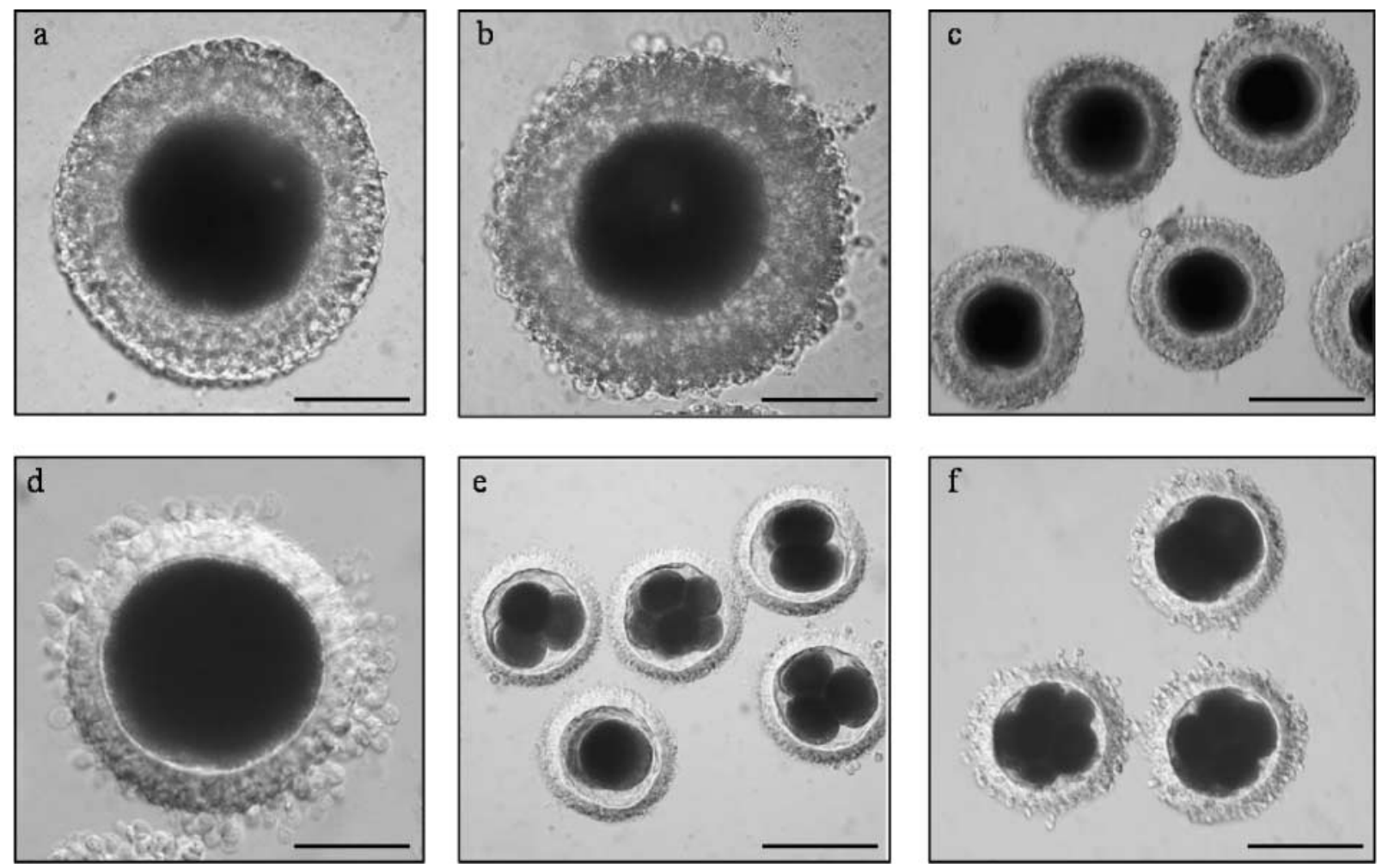

Figure 1 Canine oocytes and embryos observed under light microscopy at different stages after ovulation. Maturation stages were determined by confocal microscopy. (a) Cumulus-oocyte complex at (a) $35 \mathrm{~h}$ post-ovulation and (b) $69 \mathrm{~h}$ post-ovulation, (c) fertilized $\mathrm{Ml}$ oocytes at $72 \mathrm{~h}$ postovulation, (d) two-pronuclei embryo at $110 \mathrm{~h}$ post-ovulation and (e and f) two-, four- and eight-cell embryos ( $112 \mathrm{~h}$ and $138 \mathrm{~h}$ post-ovulation respectively). Scale bars represent (a, b and d) $50 \mu \mathrm{m}$ and (c, e and f) $100 \mu \mathrm{m}$.

Until $44 \mathrm{~h}$ after ovulation, all oocytes were at the GV stage, with the nucleus peripherally located. Surprisingly, during this post-ovulatory period, the appearance of germinal vesicles was singular. Nuclear membrane, still unfolded and continuous, delimits a space of around $20 \mu \mathrm{m}$ in diameter but DNA was restricted to much smaller zona of $4-5 \mu \mathrm{m}$ with chromatin organized around nucleoli (Fig. 2 a-c). Only one GV (out of 82) presented a classical large round-shaped structure $(21 \mu \mathrm{m}$ in diameter), with the DNA dispersed through the whole volume.

After $44 \mathrm{~h}$ post-ovulation, some GV oocytes were still present, but later stages began to be observed. No GVBD was seen at any time, $\mathrm{Ml}$ oocytes were detected starting from $48 \mathrm{~h}$ (Fig. 2d) and the first MIl oocyte (Fig. 2g) at $54 \mathrm{~h}$. Pronuclear stage embryos appeared in general after $92 \mathrm{~h}$ (Fig. $2 \mathrm{~h}$ and i). Two-cell embryos could be observed from $112 \mathrm{~h}$ (Figs $1 \mathrm{~d}$ and $2 \mathrm{j}$ ), together with embryos at the four- and eight-cell stage (Figs $1 \mathrm{e}-\mathrm{f}$ and $2 \mathrm{k}$ and $\mathrm{I}$ ).

It was clear that maturation was very homogenous before $44 \mathrm{~h}$, since all oocytes (51) from 12 bitches were at the GV stage. However, as early as meiosis resumed, cohorts began to diverge. GV, MI, TI and MII stages could be simultaneously observed in the same bitch (Fig. 3, bitch 11). Later, after fertilization, this heterogeneity persisted, as one bitch could have, at the same time, two-, three-, four- and eight-cell embryonic stages (Fig. 3, bitch 30). As a consequence, one maturation stage could be observed over a long period (GV from 17 to $109 \mathrm{~h}$ postovulation, MI from 48 to $127 \mathrm{~h}$ and MII from 54 to $127 \mathrm{~h}$ ). However, no significant influence of bitch breed and age on maturation and developmental kinetics was observed.

Figure 2 (a) Semi-thin section of a canine oocyte collected $18 \mathrm{~h}$ after ovulation. (b) Lamin (green) and DNA (red) staining of the nucleus of an oocyte collected $42 \mathrm{~h}$ after ovulation. DNA (red) and $\alpha$-tubulin (green) staining in canine oocytes/embryos followed by confocal microscopy. (c) The oocyte, $30 \mathrm{~h}$ after ovulation, presents a germinal vesicle, peripherally located, containing uncondensed DNA organized around small nucleoli. Ml oocytes collected (d) $66 \mathrm{~h}$ and (e and f) $72 \mathrm{~h}$ after ovulation and (g) an MIl oocyte collected $79 \mathrm{~h}$ after ovulation showing highly condensed, aligned chromosomes forming the metaphase plate (pb, polar body). Inserts ( $d, e, g)$; detail of the gamete DNA. Sperm penetration was observed in the cytoplasm of three immature oocytes: (e and f) slightly decondensed sperm head (sh) was visible at the vicinity of the MI plate. $90 \mathrm{~h}$ post-ovulation, embryonic stages were also observed. ( $\mathrm{h}$ and i) Two-pronuclei stage $110 \mathrm{~h}$ after ovulation, (j) two-cell stage $127 \mathrm{~h}$ after ovulation and ( $k$ and $\mathrm{l}$ ) four- and eight-cell stage collected $112 \mathrm{~h}$ after ovulation. Scale bars represent ( $\mathrm{f}$ and i) $2.5 \mu \mathrm{m}$, (b) $10 \mu \mathrm{m}$ and (a, c, d, e, g, h and j-l) $20 \mu \mathrm{m}$. 

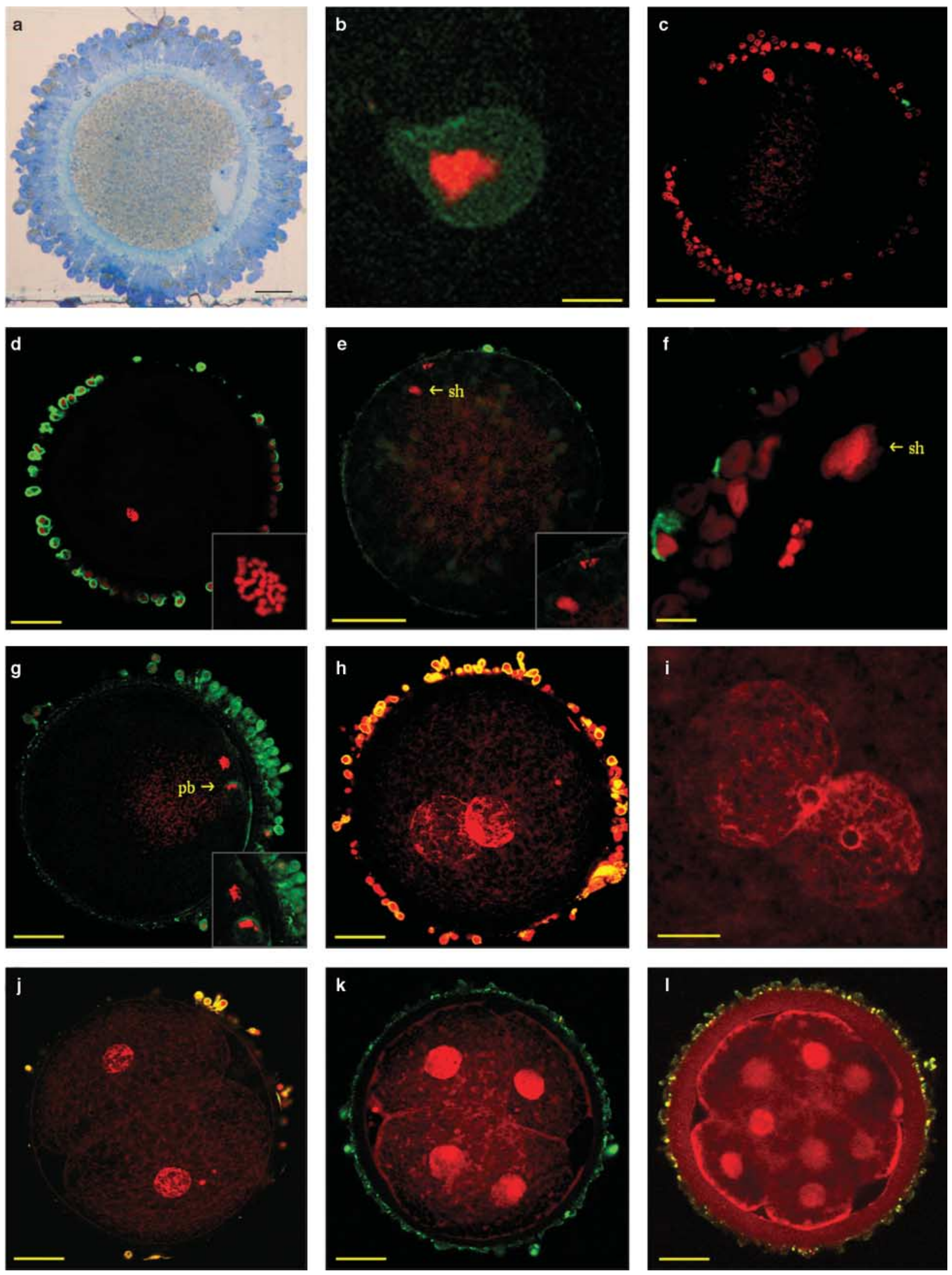


\section{Oocyte maturation stage at sperm penetration}

Using confocal microscopy, the presence of sperm heads in oocyte cytoplasm could be investigated accurately. Despite the fact that bitches were inseminated during the period ranging from the day before ovulation to surgery, we did not observe any sperm penetration in GV oocytes ( $n=82 ; 21$ bitches). Of $30 \mathrm{Ml} / \mathrm{TI}$ oocytes examined, only three were fertilized (one bitch out of 12) (Fig. 2f). Of a total of 112 immature oocytes (30 bitches), sperm penetration in immature oocytes (GV-TI stages) occurred only in one bitch, at the Ml stage, at $72 \mathrm{~h}$ after ovulation.

Interestingly, despite the presence of spermatozoa in the oviduct, sperm penetration was not observed in $90 \%$ MII oocytes present from 54 to $83 \mathrm{~h}$ after ovulation $(n=29$; nine bitches out of ten). Fertilization occurred generally from $90 \mathrm{~h}$ after ovulation.

Polyspermy was not observed whatever the oocyte stage.

\section{Discussion}

Oocyte maturation and embryonic development in canids follow a number of specific characteristics with respect to timing, site and duration. However, data available in the literature are scarce and still unclear. Most studies, in both the fox and the dog, have been conducted on very few females (from six to 25) over a very large period of time (from 0 to 24 days after ovulation) (Doak et al. 1967, Tsutsui 1975, Tsutsui \& Shimizu 1975, Renton et al. 1991, Bysted et al. 2001). Furthermore, the timing of maturation and embryonic development has been described with reference to several events more or less timely related to ovulation: onset of oestrus, acceptance of the male, LH peak, aspect of follicles at laparotomy or serum progesterone levels (see for example Tsutsui 1975, 1989, Archbald et al. 1980, Renton et al. 1991, Bysted et al. 2001). No study has been conducted with real visualization of ovulation, which appears to be the best reference for the description of oocyte maturation and embryo development. In our present work, oocytes/embryos of 50 bitches were collected during a restricted period of time covering $121 \mathrm{~h}$ soon after ovulation. Moreover, ovarian ultrasonography was used to assess the occurrence of ovulation precisely. This method has been proven to be accurate for the detection of ovulation (Boyd et al. 1993, Marseloo et al. 2004). Moreover, in contrast to laparotomy (Tsutsui 1975), ultrasonography is a non-invasive technique, respecting the welfare of the animal and inducing less stress which may disturb the LH peak and ovulation. Finally, our observations are physiologically more relevant as oocytes/embryos were collected during spontaneous natural oestrus without any hormonal manipulation such as induction of oestrus or superovulation.

Another bias in most studies focusing on canine oocyte/embryos is the high rate of undetermined nuclear stages which is due to the darkness of the oocyte cytoplasm, rich in lipid droplets. Moreover, the occurence of sperm penetration is nearly impossible to assess with conventional methods. To overcome this difficulty, we set up and validated an appropriate staining method coupled with confocal laser scanning microscopy. Observation with confocal microscopy made it possible to reduce the rate of oocytes/embryos with non-determined nuclear stage to a minimum (3\% versus about $30-40 \%$ with optic microscopy; Saint-Dizier et al. 2004).

In our study, the mean number of corpora lutea per bitch was slightly higher than that observed classically (Doak et al. 1967, Tsutsui \& Shimizu 1975, Shimizu et al. 1990, Renton et al. 1991, Bysted et al. 2001).

Oocytes/embryos were collected between 17 and $138 \mathrm{~h}$ (5.5 days) after ovulation and all of them were found in the oviduct: entry in the proximal part of the uterus has been described to occur between 168 and $240 \mathrm{~h}$ after ovulation (16- and 32-cell, morula or blastocyst stage) (Tsutsui 1975, Harper 1988, Renton et al. 1991).

Our recovery rate $(73 \%)$ was similar to that obtained by others, ranging from 40 to $99 \%$ (Tsutsui 1975 , Tsutsui \& Shimizu 1975, Renton et al. 1991, Bysted et al. 2001). In two cases, we collected respectively one and two structures more than the number of corpora lutea counted at macroscopic examination. Bysted et al. (2001) also reported this observation in one bitch out of nine. This can be explained either by ovulation of polyovular follicles (Andersen \& Simpson 1973, Telfer \& Gosden 1987) or by one (or two) corpus luteum being not apparent at the surface of the ovary.

In the dog, oocyte maturation is well known to be delayed 2-3 days after ovulation (Holst \& Phemister 1971, Tsutsui 1975) and embryonic development is much slower than in other species (for review see Betteridge 1995). However, the exact timing of meiosis during the first $48 \mathrm{~h}$ is not well described. In our study, 56 oocytes from 13 bitches were analyzed between 17 and $48 \mathrm{~h}$ after ovulation. The oocytes were found to be at the GV stage up to $44 \mathrm{~h}$, reached $\mathrm{Ml}$ between 44 and $48 \mathrm{~h}$ and the first MII appeared at $54 \mathrm{~h}$. This is in agreement with results obtained by Tsutsui (1975) reporting that MII oocytes can be observed between $48 \mathrm{~h}$ and $72 \mathrm{~h}$ (also, in this study, the time of ovulation was determined with a precision of only $24 \mathrm{~h}$ ). No GVBD was observed in our study, or in those of Farstad et al. (1993) and Tsutsui (1975). This is probably because of the short duration of this phase in vivo ( $1 \mathrm{~h}$ in most species; Sirard et al. 1989, Taieb et al. 1997) and, in the case of Tsutsui (1975), the examination technique. On the contrary, the presence of other maturation stages (GV, $\mathrm{MI}$ and MII) is spread over 70 to $90 \mathrm{~h}$ in our study (Fig. 3). However, it is impossible to discriminate between normal developing oocytes and blocked degenerating oocytes.

Interestingly, the GV aspect that we observed in these in vivo collected canine oocytes was very different; first, from the one observed in other mammalian species and, secondly, from the structure observed in in vitro cultured canine oocytes (Hewitt et al. 1998, Saint-Dizier et al. 


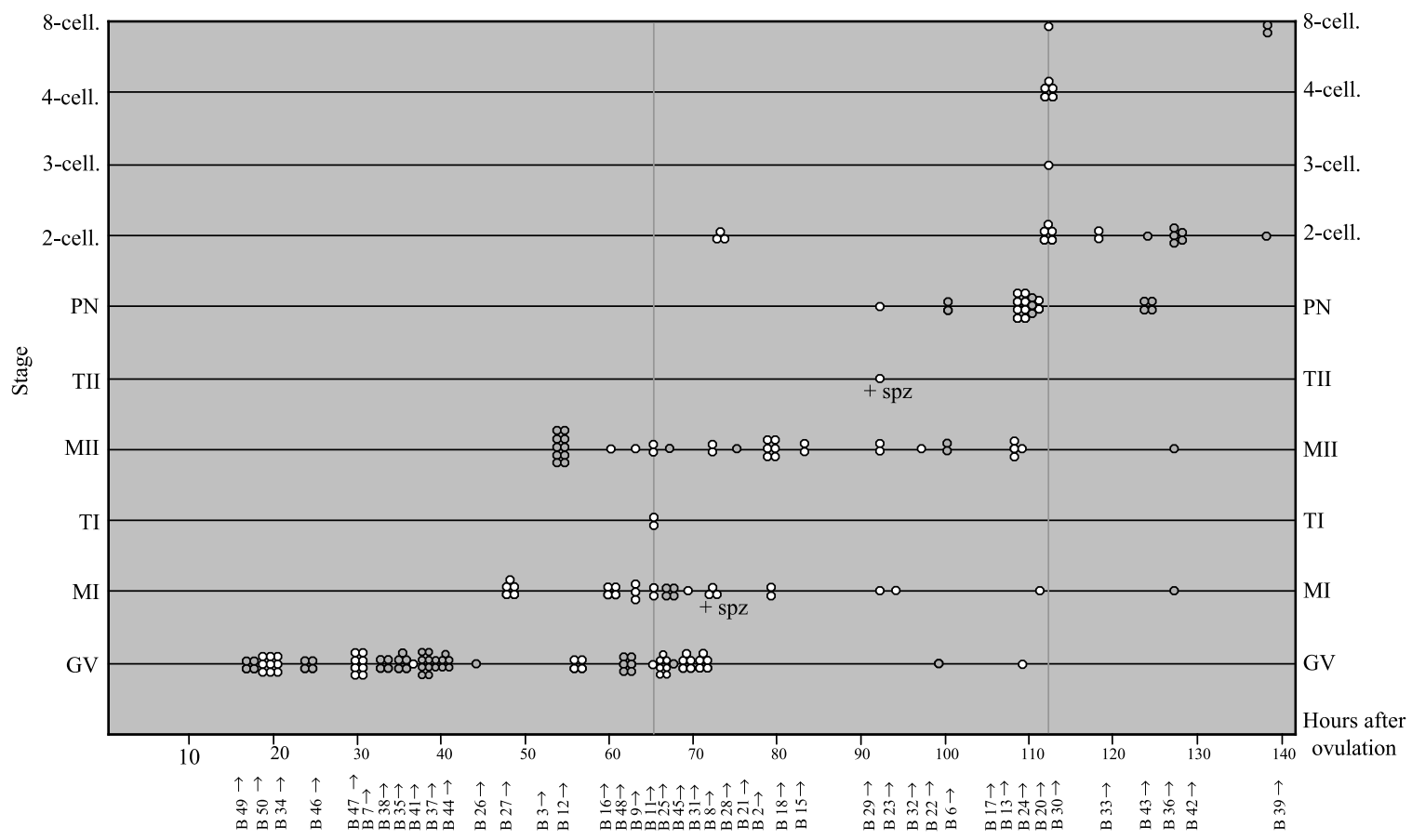

Figure 3 Timing of oocyte meiotic maturation and embryo development in reference to ovulation. Bitches were ovariectomized from 17 to $138 \mathrm{~h}$ post-ovulation. Collected oocytes/embryos were stained for observation under confocal microscopy to determine their nuclear stage (GV oocyte to eight-cell embryo). PN, two-pronuclei and + spz, presence of sperm head in oocyte cytoplasm. Heterogeneity of the developmental stages is more visible on bitches (B) 11 and 30 (vertical lines). Beagle bitches (shaded circles) and other breeds and mongrels (open circles).

2004). Lamin and semi-thin sections showed the persistance of the nuclear membrane and nucleoli were still present, but surrounded by the DNA. Some GV of the in vivo collected oocytes drawn by Van der Stricht (1923) also showed this particular aspect.

This kind of structure seems similar to the surrounded nucleolus observed in mouse and human oocytes, this configuration being thought to represent a transitional stage of GV towards ovulation (Bouniol-Baly et al. 1999, Miyara et al. 2003).

All the collected oocytes presented two or three layers of dense, compact granulosa cells, without any visible mucification as described by Andersen \& Simpson (1973) who described one oocyte in an histological section of an oviduct. No mucified masses were observed in the flushing liquid,. However, in preovulatory follicles, after the LH peak, some mucification occurs in peripheral granulosa layers surrounding the three internal dense layers (authors' unpublished data). Since granulosa cells have been shown to be responsible for the inhibition of meiosis resumption (Whitaker 1996), the persistence of close relations between the oocyte and the corona radiata after the LH peak may contribute to the delay between ovulation and meiotic resumption in the bitch.

Our protocol ensured a constant presence of spermatozoa in the oviduct starting before ovulation and until oocyte/embryo collection. Fertilization was thus possible precociously as this event is physiologically possible in vivo in the bitch. However, penetration of spermatozoa in immature oocytes was only exceptionally observed in our study. In the fox, Farstad et al. (1993) reported the opposite for in vivo sperm penetration in $46 \%$ of immature oocytes. In vitro, fertilization of immature oocytes in the bitch is a frequent event $(11-46 \%$ minimum; Yamada et al. 1992, Nickson et al. 1993, Saint-Dizier et al. 2001), but finally probably artefactual. Sperm penetration of immature oocytes can indeed be achieved in vitro in species in which ovulation occurs in vivo at the MII stage (mouse: Iwamatsu \& Chang 1975, cow: Chian et al. 1992, human: Van Blerkom et al. 1994).

Our study has clearly demonstrated that fertilization in the bitch occurs in vivo at the MIl stage. However, a striking feature was the observation of a delay in sperm penetration in mature oocytes. Despite the presence of both MII oocytes and spermatozoa for several hours, fertilization was delayed at least up to $83 \mathrm{~h}$ after ovulation, suggesting the need for a minimum period in the oviduct before fertilization. The determination of the exact time of fertilization is of clinical interest for reproductive biotechnologies and especially for artificial insemination with frozen semen, because of its short lifespan. Two-pronuclei embryos were first observed in our study $92 \mathrm{~h}$ after ovulation. At that time, oocytes are in the distal part of the oviduct (Tsutsui 1975), in contrast to other mammals, in which fertilization generally occurs in the proximal part (Harper 1988). Formation of ampulla was never observed. 
In vivo fertilization appears to be a very efficient phenomenon, with sperm penetration in $81 \%$ of fertilizable oocytes. Polyspermy, observed at a high frequency after in vitro oocyte maturation (6-59\%; Yamada et al. 1992, Otoi et al. 2000, Saint-Dizier et al. 2001), did not seem to occur in vivo. Suboptimal oocyte culture conditions before fertilization and excessive sperm concentration in the fertilization medium may be responsible for in vitro polyspermy.

Very few precise data on embryonic development timing are available in the bitch, because most studies refer to an imprecise 'starting point' such as the onset of the heat period or the acceptance of the male. The other limiting factors are the restricted number of bitches analysed at each time and the large interval (usually $24 \mathrm{~h}$ ) between two observations. With reference to ovulation detected by ultrasonography, we observed two-pronuclei stages between 92 and $124 \mathrm{~h}$, in accordance with Bysted et al. (2001) but in contrast to Tsutsui (1975) and Renton et al. (1991) who described this stage between 72 and $96 \mathrm{~h}$. In our study, two-cell embryos were present between 114 and $138 \mathrm{~h}$, and three- to four-cell embryos from $112 \mathrm{~h}$ onwards, which agrees with Tsutsui (1975) and Bysted et al. (2001). We observed eight-cell stages at a slightly earlier time than previous studies.

Heterogeneity of oocyte maturation and embryo developmental stages was obvious between the oocytes/embryos of the same cohort. This was also observed by Renton et al. (1991) and may be partially related to the asynchrony of follicle ovulation. This phenomenon can be spread out over $12 \mathrm{~h}$ (Boyd et al. 1993), leading to some diversity between oocytes.

The precise knowledge of oocyte maturation timing and early embryonic development is essential in the better understanding of developmental physiology in the bitch and consequently for the improvement of reproductive biotechnology efficiency in canids. The most striking feature of these events is the delay in the appearance of fertilizable oocyte. The precise determination of its duration is of great interest for artificial insemination with frozen semen and embryo transfer. However, further studies are necessary to identify the factors responsible for the delay in meiotic resumption in bitch oocyte.

\section{Acknowledgements}

We are grateful to Andrew Ponter, Jean-Paul Mialot and Nathalie Beaujean for critical reading of the manuscript and to Pascale Debey for her advice concerning the appearance of GV. We also wish to express our gratitude to the staff at the Centre d'Etudes en Reproduction des Carnivores, Alfort National Veterinary College for oestrus follow-up. Thanks are also due to the team who take care of the animals. The authors declare that there is no conflict of interest that would prejudice the impartiality of this scientific work.

\section{References}

Adenot PG, Szollosi MS, Chesne P, Chastant S \& Renard JP 1997 In vivo aging of oocytes influences the behavior of nuclei transferred to enucleated rabbit oocytes. Molecular Reproduction and Development 46 325-336.

Andersen AC \& Simpson ME 1973 Puberty-The first oestrus cycle in pregnant and non-pregnant Beagles. In The Ovary and Reproductive Cycle of the Dog (Beagle). Chapter IV, pp 105-127. Los Altos, CA: Geron-X Inc.

Andersen K 1975 Insemination with frozen dog semen based on a new insemination technique. Zuchthygiene 10 1-4.

Archbald LF, Baker BA, Clooney LL \& Godke RA 1980 A surgical method for collecting canine embryos after induction of estrus and ovulation with exogenous gonadotropins. Veterinary Medicine Small Animal Clinician 75 228-238.

Betteridge KJ 1995 Phylogeny, ontogeny and embryo transfer. Theriogenology 44 1061-1098.

Boyd JS, Renton JP, Harvey MJ, Nickson DA, Eckersall PD \& Ferguson JM 1993 Problems associated with ultrasonography of the canine ovary around the time of ovulation. Journal of Reproduction and Fertility 47 (Suppl) 101-105.

Bysted BV, Dieleman SJ, Hyttel P \& Greve T 2001 Embryonic developmental stages in relation to the LH peak in dogs. Journal of Reproduction and Fertility 57 (Suppl) 181-186.

Chian RC, Niwa K \& Nakahara H 1992 Effect of sperm penetration in vitro on completion of first meiosis by bovine oocytes arrested at various stages in culture. Journal of Reproduction and Fertility 96 $73-78$.

Doak RL, Hall A \& Dale HE 1967 Longevity of spermatozoa in the reproductive tract of the bitch. Journal of Reproduction and Fertility 13 51-58.

Farstad W, Hyttel P, Grondahl C, Mondain-Monval M \& Smith AJ 1993 Fertilization and early embryonic development in the blue fox (Alopex lagopus). Molecular Reproduction and Development $36331-337$.

Harper MJK 1988 Gamete and zygote transport. In The Physiology of Reproduction, pp 103-134. Eds E Knobil \& JD Neill.

Hayer P, Gunzel-Apel AR, Luerssen D \& Hoppen HO 1993 Ultrasonographic monitoring of follicular development, ovulation and the early luteal phase in the bitch. Journal of Reproduction and Fertility 47 (Suppl) 93-100.

Hewitt DA, Watson PF \& England GC 1998 Nuclear staining and culture requirements for in vitro maturation of domestic bitch oocytes. Theriogenology 49 1083-1101.

Holst PA \& Phemister RD 1971 The prenatal development of the dog: preimplantation events. Biology of Reproduction 5 194-206.

Iwamatsu T \& Chang MC 1975 Sperm penetration in vitro of mouse oocytes at various times during maturation. Journal of Reproduction and Fertility 31 237-247.

Marseloo N, Fontbonne A, Bassu G, Riviere S, Leblanc B, Rault D, Biourge V \& Chastant-Maillard S 2004 Comparison of ovarian ultrasonography with hormonal parameters for the determination of the time of ovulation in bitches. In Proceedings of the 5th International Symposium on Canine and Feline Reproduction, pp 75-77. Sao Paulo, Brasil.

Miyara F, Migne C, Dumont-Hassan M, Le Meur A, Cohen-Bacrie P Aubriot FX, Glissant A, Nathan C, Douard S, Stanovici A \& Debey P 2003 Chromatin configuration and transcriptional control in human and mouse oocytes. Molecular Reproduction and Development 64 458-470.

Nickson DA, Boyd JS, Eckersall PD, Ferguson JM, Harvey MJ \& Renton JP 1993 Molecular biological methods for monitoring oocyte maturation and in vitro fertilization in bitches. Journal of Reproduction and Fertility 47 (Suppl) 231-240.

Otoi T, Fujii M, Tanaka M, Ooka A \& Suzuki T 2000 Oocyte diameter in relation to meiotic competence and sperm penetration. Theriogenology 54 535-542. 
Renton JP, Boyd JS, Eckersall PD, Ferguson JM, Harvey MJ, Mullaney J \& Perry B 1991 Ovulation, fertilization and early embryonic development in the bitch (Canis familiaris). Journal of Reproduction and Fertility 93 221-231.

Reynaud K, Saint-Dizier M \& Chastant-Maillard S 2004 In vitro maturation and fertilization of canine oocytes. In Methods in Molecular Biology, Germ Cell Protocoles. Volume 1: Sperm and Oocyte Analysis, pp 255-272. Ed. H Schatten. USA: Humana Press Inc.

Saint-Dizier M, Renard JP \& Chastant-Maillard S 2001 Induction of final maturation by sperm penetration in canine oocytes. Reproduction 121 97-105.

Saint-Dizier M, Reynaud K \& Chastant-Maillard S 2004 Chromatin, microtubules, and kinases activities during meiotic resumption in bitch oocytes. Molecular Reproduction and development $\mathbf{6 8}$ 205-212.

SAS 1992 In Technical Report, release 6.07 Cary, NC: SAS Institute Inc.

Shimizu T, Tsutsui T, Murao I \& Orima H 1990 Incidence for transuterine migration of embryos in the dog. Japanese Journal of Veterinary Science 52 1273-1275.

Sirard MA, Florman HM, Leibfried-Rutledge ML, Barnes FL, Sims ML \& First NL 1989 Timing of nuclear progression and protein synthesis necessary for meiotic maturation of bovine oocytes. Biology of Reproduction 40 1257-1263.

Taieb R, Thibier C \& Jessus C 1997 On cyclins, oocytes, and eggs. Molecular Reproduction and Development 48 397-411.

Telfer E \& Gosden RG 1987 A quantitative cytological study of polyovular follicles in mammalian ovaries with particular reference to the domestic bitch (Canis familiaris). Journal of Reproduction and Fertility 81 137-147.

Tsutsui T 1975 Studies on the reproduction in the dog. V. On cleavage and transport of fertilized ova in the oviduct. Japanese Journal of Animal Reproduction 21 70-75.

Tsutsui T 1989 Gamete physiology and timing of ovulation and fertilization in dogs. Journal of Reproduction and Fertility 39 (Suppl) 269-275.

Tsutsui T \& Shimizu T 1975 Studies on the reproduction in the dog IV. On the fertile period of ovum after ovulation. Japanese Journal of Animal Reproduction 21 65-69.

Van Blerkom J, Davis PW \& Merriam J 1994 The developmental ability of human oocytes penetrated at the germinal vesicle stage after insemination in vitro. Human Reproduction 9 697-708.

Van der Stricht O 1923 Etude comparée des ovules de mammifères aux différentes périodes de l'ovogenèse. Archives de Biologie 33 229-300.

Yamada S, Shimazu Y, Kawaji H, Nakazawa M, Naito K \& Toyoda Y 1992 Maturation, fertilization, and development of dog oocytes in vitro. Biology of Reproduction 46 853-858.

Whitaker M 1996 Control of meiotic arrest. Reviews of Reproduction 1 127-135.

Received 28 September 2004

First decision 1 November 2004

Revised manuscript received 1 April 2005

Accepted 10 May 2005 